\title{
Predictive Control for Longitudinal Beam Dynamics in Heavy Ion Synchrotrons
}

\author{
Timm Faulwasser ${ }^{1}$, Dieter Lens ${ }^{2}$ and Christopher M. Kellett ${ }^{3}$
}

\begin{abstract}
We present a design of a predictive control scheme for longitudinal beam dynamics in heavy ion synchrotrons. Specifically, we consider a linear-quadratic model predictive control (MPC) approach, whereby the quadratic program is solved via a fast gradient method. Furthermore, we investigate whether the fast gradient method allows for real-time feasible implementation of the proposed approach on a field programmable gate array (FPGA). Our results indicate that sampling rates in the order of $1 \mathrm{MHz}$ are achievable.

Index Terms-model predictive control, heavy ion synchrotrons, longitudinal beam control, fast gradient method, field programmable gate array
\end{abstract}

\section{INTRODUCTION}

Particle accelerators such as synchrotrons are frequently used for research purposes in particle physics and medical applications. Due to their nonlinear and fast dynamics the operation of synchrotrons poses interesting control-specific challenges. For example, consider modelling the dynamic behavior of highly accelerated particle bunches in synchrotrons or the task to control a particle bunch with respect to its shape in the longitudinal phase space.

In this paper we investigate whether state-of-the-art model predictive control (MPC) schemes can be applied to particle beam control problems in synchrotrons. Synchrotrons are mostly located in research institutions and used for scientific purposes. This implies that hardware used for control purposes might differ significantly from synchrotron to synchrotron. Here, we focus on the heavy-ion synchrotron SIS18, which is used at the GSI Helmholtz Centre for Heavy Ion Research in Darmstadt, Germany [6]. A main challenge of applying MPC to synchrotrons is that the required sample rates are in the order of $100-1000 \mathrm{kHz}$. While this is quite fast for numerically intensive control schemes, such as MPC, the control architecture of the SIS18 synchrotron allows implementation of the control algorithms on a field programmable gate array (FPGA).

Due to the very fast dynamics of beam oscillations in hadron (i.e. proton and heavy-ion) synchrotrons, only analog and digital filters or PI controllers have been used in these

\footnotetext{
$1 \mathrm{TF}$ is with the Laboratoire d'Automatique, Ecole Polytechnique Fédérale de Lausanne, $\mathrm{CH}-1004$ Lausanne, Switzerland timm. faulwasser@epfl.ch

2 DL is with the Institut für Automatisierungstechnik, Technische Universität Darmstadt, D-64283 Darmstadt, Germany dlensertr.tu-darmstadt.de

$3 \mathrm{CK}$ is with the School of Electrical Engineering and Computer Science, University of Newcastle, Callaghan NSW 2308 Australia chris.kellett@newcastle.edu.au. CK is supported by the Australian Research Council under FT1101000746. This work was also partially supported by the Alexander von Humboldt Foundation, Germany.
}

accelerators to date $[2,8,10]$. The main motivation to consider MPC for longitudinal beam stabilization in hadron synchrotron machines is twofold: (1) MPC is a structured state-feedback control approach, which should allow to further improve the control performance and therefore the beam quality compared to existing designs, enabling better experimental conditions; and (2) MPC allows to consider input and state constraints. Due to several technical and physical reasons such as limited power and bandwidth of the RF cavities used to control the beam, input constraints should be considered for longitudinal beam stabilization in hadron synchrotrons. To the best of the authors' knowledge, modern model-based control techniques such as MPC have never been used for longitudinal beam stabilization in any hadron synchrotron machine.

For electron synchrotrons, which exhibit significantly different behavior, this observation seems to be equally true [1, 13, 22]. For the transverse orbit correction, the references [4, 17] present results on transverse orbit stabilization. The sample rate time is given as $10 \mathrm{kHz}$, which is considerably slower than the sampling times considered in this paper.

In [3], for electron synchrotrons, it is stated that due to the large dimension of the system and the fast sampling rate, MPC is not feasible. For the heavy-ion synchrotron SIS18 at GSI, the situation is different: the upgrade of the digital feedback systems as part of the FAIR project (cf. [24]) will include modern high speed FPGAs. The aim of this paper is to show that MPC is feasible in principle for SIS18, although the sample rate is about $375 \mathrm{kHz}$. In [5], the SIS18 synchrotron serves as an example for a robust observer based state feedback controller, but no implementation and timing issues are discussed. In this work, we aim to make a considerable step towards the implementation of MPC on digital hardware for heavy-ion synchrotrons.

The implementation of MPC on FPGAs was previously investigated in $[7,9,16,25]$. While $[7,16]$ use interior point algorithms, other results consider active set strategies [25] to solve the arising quadratic program. Recently, the use of fast gradient methods (FGM) for MPC on embedded platforms $[12,21]$ and on FPGAs [9] has also been investigated. A major advantage of the FGM compared to interior-point or active set methods is that it does not involve the solution of a linear system of equations. It merely requires vectormatrix multiplications and additions. However, in the FGM only input constraints with a rather simple geometry can be considered and handling state constraints is in general challenging.

While [9] focussed on the derivation of error bounds and 
convergence guarantees for fixed-point implementations of the FGM, our focus lies on assessing whether an FGMbased MPC scheme can be used for control problems which arise in synchrotron operation. Specifically, we consider the problem of longitudinal beam feedback control in heavy ion synchrotrons. In contrast to [9], which considers a partially parallelized multi-cycle implementation, we focus on a completely parallelized single-cycle design for the implementation of the FGM on an FPGA. Furthermore, we provide preliminary timing analysis results for a specific FPGA technology, which indicates that particle beam control problems can be tackled with FPGA-based MPC.

The remainder of the paper is structured as follows: In Section II we will concisely present how the dynamic behavior of particle bunches in synchrotrons can be modeled. This modeling will lead to a formulation of the longitudinal beam feedback control problem. Section III describes the proposed MPC scheme and presents results obtained from a simulation study. Section IV provides insight into the sampling rates, which are achievable assuming the proposed MPC controller is implemented on a high-end FPGA such as a Stratix V from Altera.

\section{PARTICLE BEAM DYNAMICS}

\section{A. Nonlinear Modeling}

A hadron synchrotron is a ring accelerator with a closed reference orbit that is used to accelerate protons and ions to high energies [14]. A simplified scheme is shown in Fig. 1. The magnetic fields of the dipole and quadrupole magnets are used for the transverse focussing of the particle beam. The beam consists of one or more particle bunches which move inside the beampipe under vacuum conditions. One or more so-called cavities are used to accelerate and focus the beam in the longitudinal direction, i.e. in direction of motion. In the following, only the longitudinal motion of the beam is considered. In many cases, it is reasonable to assume that the longitudinal dynamics are decoupled from the transverse dynamics.

If the operation of the cavity is single harmonic and the beam energy is kept constant (such as in the case of storage rings), the dynamics of a particle in the longitudinal phase plane $(\varphi, \dot{\varphi})$ are determined by

$$
\ddot{\varphi}_{j}(t)=-\omega_{\text {syn }, 0}^{2}\left(1+u_{2}(t)\right) \sin \left(\varphi_{j}(t)-u_{1}(t)+d_{2}(t)\right),
$$

where $j=1, \ldots, N_{\mathrm{p}}$ is the particle index and $\varphi_{j}$ is the phase deviation of the particle with respect to the so-called reference $\mathrm{R}$ as shown in Fig. 1. This reference can be considered an ideal moving reference trajectory on the orbit with the exact position and velocity. The phase and amplitude modulations $u_{1}$ and $u_{2}$ can be used as inputs to control the beam shape. These inputs are subject to the constraint

$$
\forall t: \quad\left(u_{1}(t), u_{2}(t)\right)^{T} \in \mathcal{U}=\left[-\bar{u}_{1}, \bar{u}_{1}\right] \times\left[-\bar{u}_{2}, \bar{u}_{2}\right] .
$$

The disturbance $d_{2}$ in (1) will be used to excite the beam to test the feedback. The resulting particle trajectories in the phase plane $(\varphi, \dot{\varphi})$ are shown in Fig. 2 (left). The

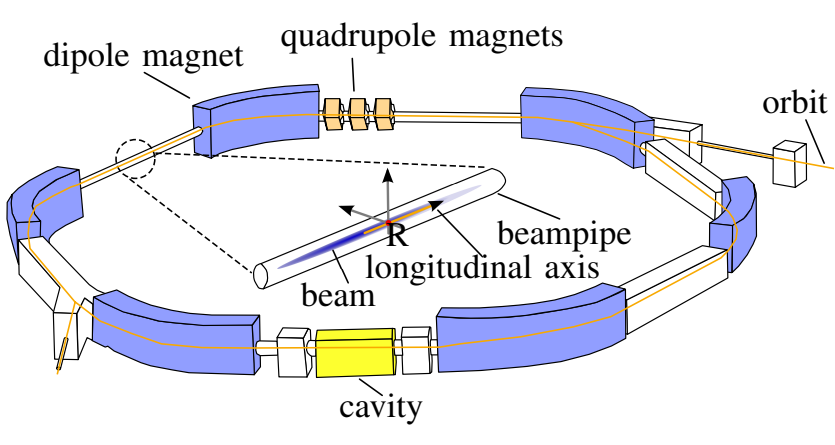

Fig. 1. Simplified setup of a synchrotron.

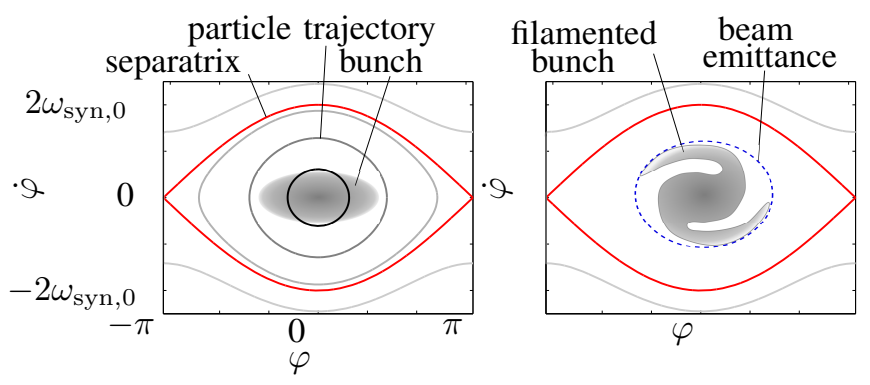

Fig. 2. Particle trajectories (left) and definition of the emittance (right).

separatrix separates stable from unstable oscillations. Inside the separatrix, the particle ensemble is usually called a bunch. The longitudinal dynamics can be derived from a Hamiltonian and the theorem of Liouville is valid, cf. [23]. One consequence is that the area occupied by the bunch in phase space is a constant of motion.

The synchrotron frequency $\omega_{\text {syn }, 0}$ is the oscillation frequency of particles near the origin $(0,0)$. The principle of filamentation is visualized in the right plot of Fig. 2. As particles with large oscillation amplitudes have a frequency smaller than $\omega_{\mathrm{syn}, 0}$, the shape of the bunch may change. Although the bunch area will remain constant in theory due to Liouville's theorem, the filamentation may be considerable and it then will be difficult to identify the actual border of the bunch shape. Thus, an effective bunch area is usually defined, the so-called emittance, cf. Fig. 2 (right). This emittance will increase in case of filamentation and one goal of the feedback will be to damp bunch oscillations to prevent this emittance blow-up as this deteriorates the beam quality. Therefore, the emittance will also be a measure for the quality of the feedback. We will formally define the emittance after the definition of the bunch moments below.

The synchrotron frequency can be calculated as

$$
\omega_{\text {syn }, 0}=\omega_{\mathrm{R}} \sqrt{\frac{h Q\left[\gamma_{\mathrm{R}}^{-2}-\gamma_{\mathrm{tr}}^{-2}\right]}{2 \pi \beta_{\mathrm{R}}^{2} W_{\mathrm{R}}}} \sqrt{\left(1+d_{1}\right) \hat{V}},
$$

where

$$
\omega_{\mathrm{R}}=\frac{2 \pi}{T_{\mathrm{R}}}
$$

is the revolution frequency of the beam in the synchrotron ring. In this paper, the setup of the beam experiment described in [15] and its corresponding parameter values will 

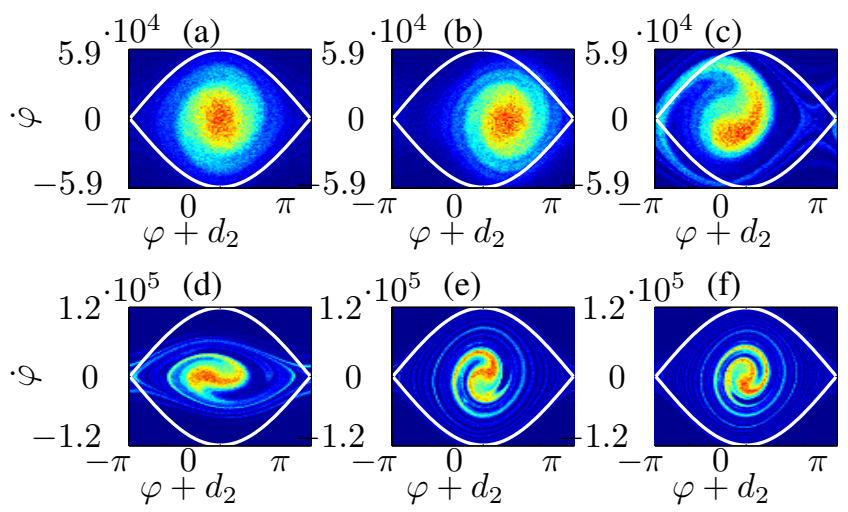

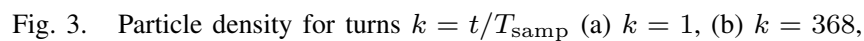
(c) $k=718$, (d) $k=1068$, (e) $k=1750$, (f) $k=2100$.

be assumed for a simulation of the feedback. See [15] for more details on the experimental setup. A revolution period $T_{\mathrm{R}}=4.6629 \mu \mathrm{s}$ will be assumed in the following. The other parameters and their values are the harmonic number $h=8$, the ion charge $Q=2.8839 \cdot 10^{-18} \mathrm{C}$, the relativistic Lorentz factors $\gamma_{\mathrm{R}}=1.0122$ and $\beta_{\mathrm{R}}=0.1550$, the transition gamma $\gamma_{\mathrm{tr}}=5.45$, the reference ion energy $W_{\mathrm{R}}=6.0349 \mathrm{~nJ}$ and the voltage amplitude $\hat{V}=5000 \mathrm{~V}$. Again, $d_{1}$ denotes a disturbance to excite beam oscillations.

As shown in [15], the bunch shape may be described by its first-order moments (mean or bunch center)

$$
\rho_{\varphi}=\frac{1}{N_{\mathrm{p}}} \sum_{j=1}^{N_{\mathrm{p}}} \varphi_{j}, \quad \rho_{\dot{\varphi}}=\frac{1}{N_{\mathrm{p}}} \sum_{j=1}^{N_{\mathrm{p}}} \dot{\varphi}_{j}
$$

and second-order moments (variances and covariance)

$$
\begin{aligned}
& \mu_{\varphi}=\frac{1}{N_{\mathrm{p}}} \sum_{j=1}^{N_{\mathrm{p}}}\left(\varphi_{j}-\rho_{\varphi}\right)^{2}, \\
& \mu_{\varphi, \dot{\varphi}}=\frac{1}{N_{\mathrm{p}}} \sum_{j=1}^{N_{\mathrm{p}}}\left(\varphi_{j}-\rho_{\varphi}\right)\left(\dot{\varphi}_{j}-\rho_{\dot{\varphi}}\right), \\
& \mu_{\dot{\varphi}}=\frac{1}{N_{\mathrm{p}}} \sum_{j=1}^{N_{\mathrm{p}}}\left(\dot{\varphi}_{j}-\rho_{\dot{\varphi}}\right)^{2} .
\end{aligned}
$$

A stationary or matched bunch has constant moments.

With the defined moments and assuming an ellipsoidal shape, the emittance can be calculated as

$$
\varepsilon_{\varphi, \dot{\varphi}}=\sqrt{\mu_{\varphi} \mu_{\dot{\varphi}}-\mu_{\varphi, \dot{\varphi}}^{2}}
$$

\section{B. Open-loop Beam Dynamics}

For the simulation of the feedback, a specific disturbance sequence will be used to excite typical beam oscillations. The sequence will be defined by

$$
d_{1}(k)=\left\{\begin{array}{ll}
3 & \text { if } k \geq k_{3} \\
0 & \text { else }
\end{array}, d_{2}(k)= \begin{cases}0.8 & \text { if } k \in\left[k_{1} ; k_{2}\right] \\
0 & \text { else }\end{cases}\right.
$$

where $k=t / T_{\text {samp }}$ is the sampling step, $T_{\text {samp }}=2.66 \mu \mathrm{s}$ the sampling time of the digital hardware containing the FPGA, and $k_{1}=368, k_{2}=718$, and $k_{3}=1068$. Disturbance $d_{2}$ will cause an excitation of the bunch center $\rho_{\varphi}$ at $k=368$ and $k=718$, whereas $d_{1}$ will excite the bunch length $\mu_{\varphi}$ at $k=1068$. The initial distribution of the particles is assumed
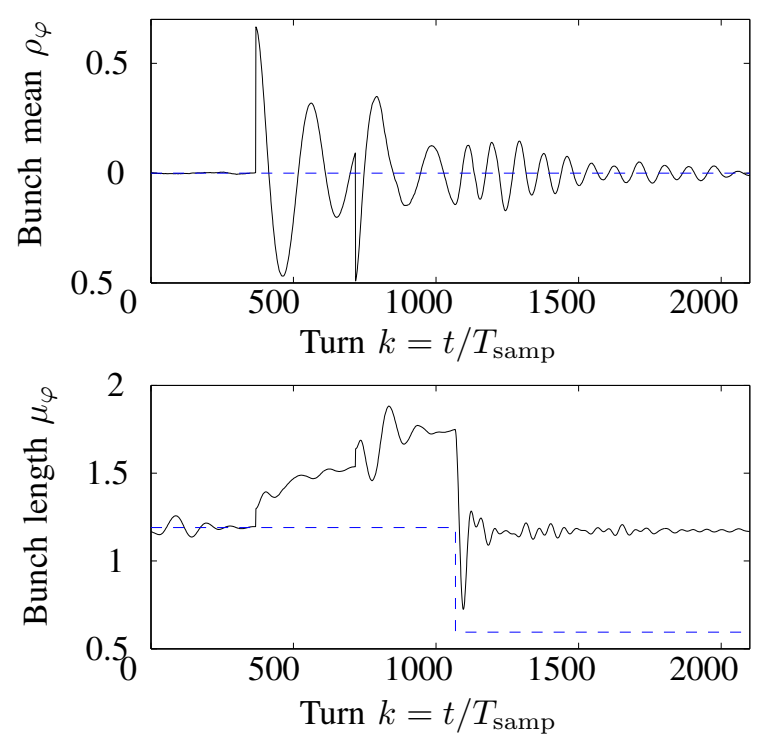

Fig. 4. Moments, open loop.

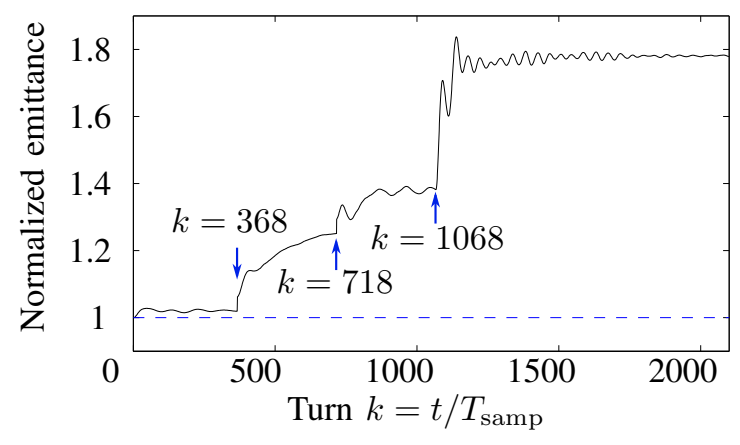

Fig. 5. Normalized emittance $\varepsilon_{\varphi, \dot{\varphi}}(k) / \varepsilon_{\varphi, \dot{\varphi}}(0)$, open loop.

to be Gaussian and matched. An open-loop simulation using these specific disturbances and with about $2.5 \cdot 10^{5}$ particles is shown in Fig. 3 for different turns.

The resulting moments are given in Fig. 4. It is apparent that the bunch is stationary until the first disturbance of $\rho_{\varphi}$ at $k=368$. This disturbance has also an effect on the bunch length, because the filamentation increases the bunch size. Since this is a highly nonlinear effect, it is practically impossible to obtain an analytical expression for this increase. The disturbance at $k=718$ again excites the mean and increases the bunch length. At $k=1068$, the voltage amplitude $\hat{V}$ is increased using $d_{1}=3$. Due to (3), the synchrotron frequency increases by a factor of $\sqrt{1+d_{1}}$ and the height of the separatrix changes by the same factor, cf. Figs. 2 and 3. This excites large bunch length oscillations, as can be seen in Fig. 4. In this figure, theoretical tangents are depicted by dashed-blue lines. These tangents represent the steady states of bunch length and bunch mean, if perfect feedback would instantly damp the oscillations and hence no filamentation would take place.

The (normalized) bunch emittance corresponding to the 
disturbance sequence (7) is shown in Fig. 5. The blue dashed line depicts again the theoretical tangent. It is apparent that every excitation increases the emittance and thus deteriorates the beam quality. At the end of the simulation, it has increased by about $80 \%$. Note that the disturbances on the beam (7) were chosen rather large to evaluate worst-case scenarios. In normal operation, the task of the feedback is to avoid smaller beam oscillations that may grow if not damped. However, also large oscillations may occur due to mismatch of the beam and this will impose the highest requirements on the feedback in terms of input constraints and possible performance issues due to model mismatch.

The conclusion from these open-loop simulations is that the ultimate goal of minimizing beam emittance can be reformulated as the problem of stabilizing the bunch moments at their setpoint. Subsequently, we consider the bunch moments (6) as controlled variables and derive a control specific model of the aggregated bunch dynamics.

\section{Derivation of a Linearized Model}

A simplified model to describe the moment dynamics has been derived in [15]. For the model, a normalized coordinate

$$
w=-\frac{1}{\omega_{\mathrm{syn}, 0}} \dot{\varphi}
$$

is used for convenience. The moments of the coordinates $(\varphi, w)$ can be easily converted into the already defined moments. The moments $\rho_{\varphi}$ and $\mu_{\varphi}$ remain the same, while the other moments read

$$
\rho_{\dot{\varphi}}=-\omega_{\mathrm{syn}, 0} \rho_{w}, \mu_{\varphi, \dot{\varphi}}=-\omega_{\mathrm{syn}, 0} \mu_{\varphi, w}, \mu_{\dot{\varphi}}=\omega_{\mathrm{syn}, 0}^{2} \mu_{w} .
$$

The model derivation involves a linearization around the setpoint

$$
\begin{aligned}
x_{\mathrm{e}} & =\left(\begin{array}{lllll}
\rho_{\varphi} & \rho_{w} & \mu_{\varphi} & \mu_{\varphi, w} & \mu_{w}
\end{array}\right)^{\mathrm{T}} \\
& =\left(\begin{array}{lllll}
0 & 0 & \bar{\mu}_{\varphi} & 0 & \bar{\mu}_{w}
\end{array}\right)^{\mathrm{T}} \\
u_{\mathrm{e}} & =\left(\begin{array}{ll}
0 & 0
\end{array}\right)^{T} .
\end{aligned}
$$

The state vector of the model is defined as

$$
x=\left(\begin{array}{lllll}
\rho_{\varphi} & \rho_{w} & \mu_{\varphi}-\bar{\mu}_{\varphi} & \mu_{\varphi, w} & \mu_{w}-\bar{\mu}_{w}
\end{array}\right)^{\mathrm{T}}
$$

and the dynamics are

$$
\dot{x}=\omega_{\mathrm{syn}, 0}\left(\begin{array}{cc}
A_{1} & 0 \\
0 & A_{2}
\end{array}\right) x+\omega_{\mathrm{syn}, 0}\left(\begin{array}{cc}
b_{1} & 0 \\
0 & b_{2}
\end{array}\right)\left(\begin{array}{l}
u_{1} \\
u_{2}
\end{array}\right),
$$

with

$$
A_{1}=\left(\begin{array}{rr}
0 & -1 \\
a_{1} & 0
\end{array}\right), A_{2}=\left(\begin{array}{crr}
0 & -2 & 0 \\
a_{2} & 0 & -1 \\
0 & a_{3} & 0
\end{array}\right)
$$

and

$$
b_{1}=\left(\begin{array}{r}
0 \\
-a_{1}
\end{array}\right), b_{2}=\left(\begin{array}{c}
0 \\
b_{1} \\
0
\end{array}\right)
$$

The model coefficients are functions of the setpoint $\bar{\mu}_{\varphi}$ :

$a_{1}=1+\sum_{n=1}^{6} \frac{(-1)^{n}}{n ! 2^{n}} \bar{\mu}_{\varphi}^{n}, \quad a_{2}=1+\sum_{n=1}^{6} \frac{(-1)^{n}(n+1)}{n ! 2^{n}} \bar{\mu}_{\varphi}^{n}$,

$a_{3}=2 a_{1}, \quad b_{1}=a_{1} \bar{\mu}_{\varphi}$,

and, for the equilibrium,

$$
\bar{\mu}_{w}=b_{1}=a_{1} \bar{\mu}_{\varphi}
$$

holds. As a conclusion, for these parameters only one degree of freedom remains and that is the bunch size setpoint $\bar{\mu}_{\varphi}$.

The longitudinal beam feedback control problem can be summarized as follows: Stabilize the bunch moments (6), which are the outputs of the nonlinear system (1), at the steady state (8) subject to the input constraint (2).

\section{PREDICTIVE PARTICLE BEAM CONTROL}

In this paper we propose to solve the longitudinal beam feedback control problem via MPC. MPC is based on the repeated solution of an optimal control problem. The problem to be solved repeatedly is given by

$$
\begin{array}{ll}
\min _{\{u(i)\}} \quad \frac{1}{2} x(N)^{T} \operatorname{Px}(N) \\
\quad+\frac{1}{2} \sum_{i=0}^{N-1} \frac{1}{2} x(i)^{T} Q x(i)+\frac{1}{2} u(i)^{T} R u(i)
\end{array}
$$

subject to $\forall i=1, \ldots, N-1$

$$
x(i+1)=A x(i)+B u(i), \quad x(0)=x_{\text {meas }}(k)
$$

and the input constraint (2). The prediction model (11b) is obtained via a zero-order-hold discretization of (9) with a sampling time $T_{\text {samp }}=2.66 \mu$ s. This sampling time is chosen in order to compare the MPC controller with a previously implemented FIR filter [15].

At each sampling step $k \in \mathbb{N}$, measured or estimated state information $x_{\text {meas }}(k)$ is obtained, the optimal control problem (11) is solved, and the first element of the optimal input sequence $\left\{u(1)^{\star}, u(2)^{\star}, \ldots, u(N-1)^{\star}\right\}$ is applied to the simulated nonlinear bunch shape dynamics (1). ${ }^{1}$

It is well-known that the optimal control problem (11) can easily be reformulated as a Quadratic Program (QP)

$$
\min _{z} \underbrace{z^{T} H z+z^{T} F x_{m e a s}(k)+c}_{J(z)}
$$

subject to

$$
z \in[-\bar{z}, \bar{z}] \subset \mathbb{R}^{\operatorname{dim}(u) \cdot N}
$$

The input sequence $\{u(0), u(1), \ldots, u(N-1)\}$ is rewritten as a vector $z=\left(u(0)^{T}, u(1)^{T}, \ldots, u(N-1)^{T}\right)^{T} \in$ $\mathbb{R}^{\operatorname{dim}(u) \cdot N}$. Positive definiteness of the penalty matrix $R$ and positive semi-definiteness of $Q$ in (11) ensure strict convexity of (12). The fast transients of the bunch shape dynamics,

\footnotetext{
${ }^{1}$ Note that the variables $\rho_{\varphi}$ and $\mu_{\varphi}$ can be computed in real-time from available process measurements. Furthermore, it is easy to verify that the linear system (9) is observable, provided that the states $x_{1}=\rho_{\varphi}$ and $x_{3}=\mu_{\varphi}$ are available.
} 

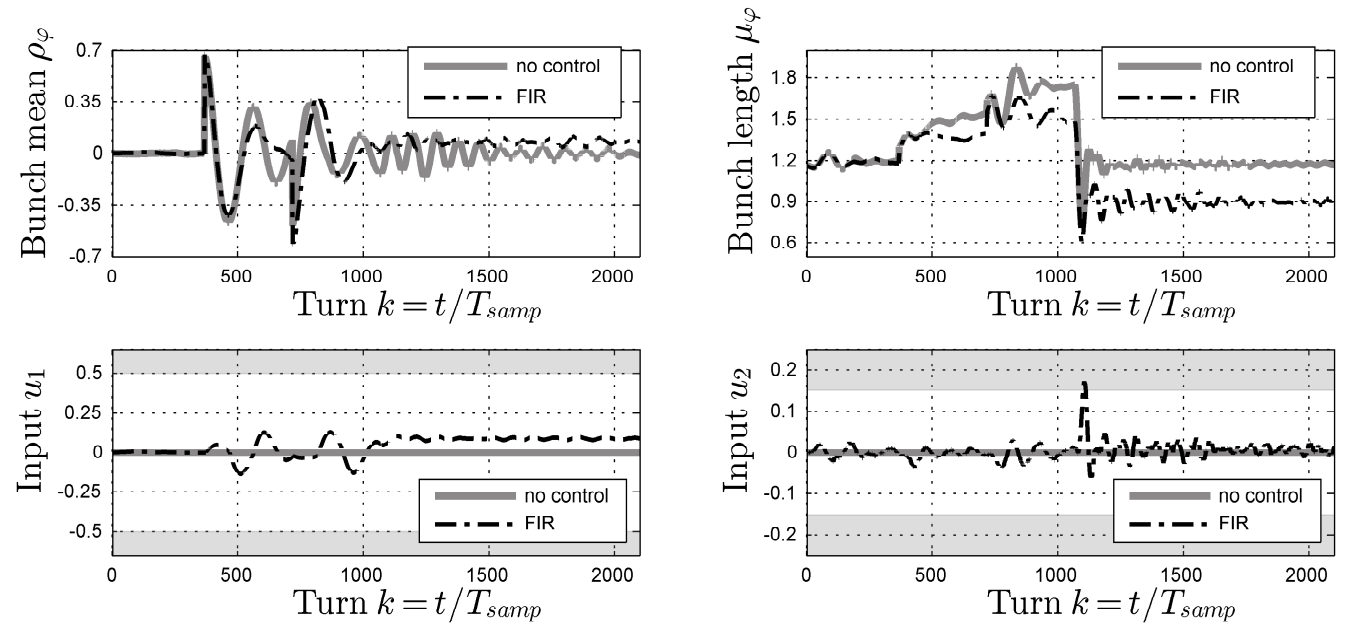

Fig. 6. Bunch mean, bunch length dynamics and corresponding inputs for open loop (black) and the FIR filter (red).
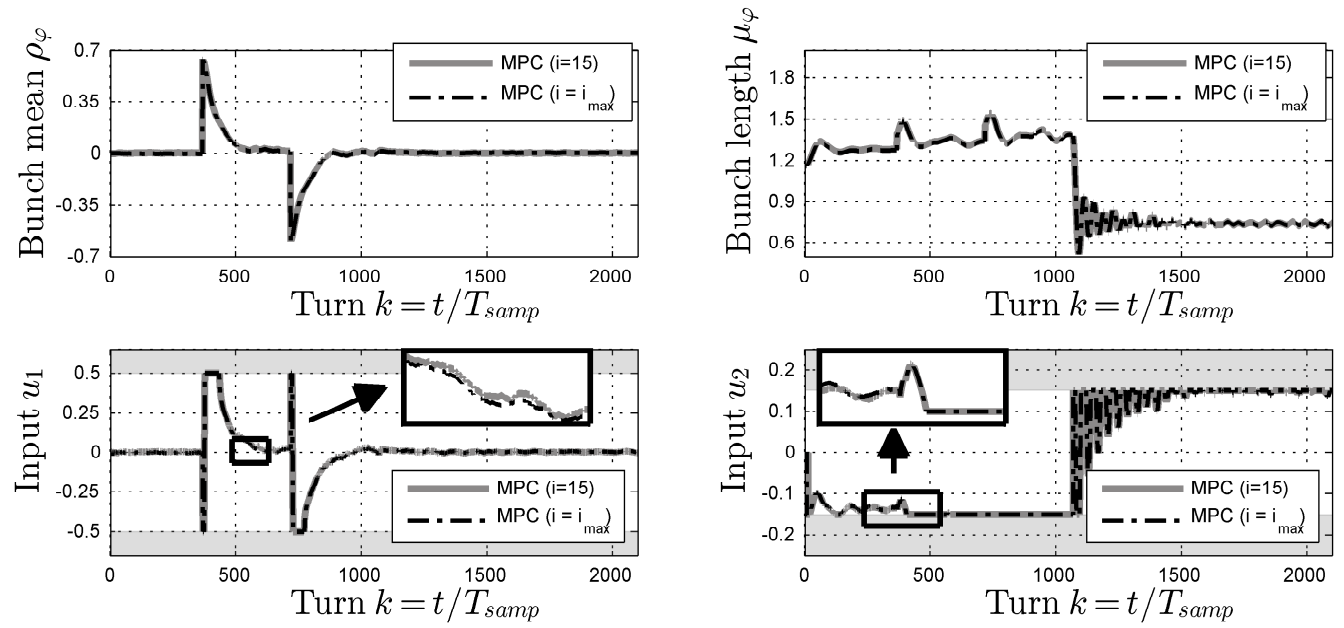

Fig. 7. Bunch mean, bunch length dynamics and corresponding inputs for MPC $(i=15)$ (blue) and MPC ( $i=33$ ) (green).

however, require a short sampling time of $T_{\text {samp }}=2.66 \mu \mathrm{s}$ for the MPC controller. Thus we consider a variant of Nesterov's Fast Gradient Method (FGM) [18], which provides a computationally efficient method to solve (12), see [12, 21, 26]. A brief description of the FGM is provided in Section IV.

\section{A. Simulation Results}

In order to assess the applicability of MPC for longitudinal beam feedback control in synchrotrons, we consider the following simulation setup: The nonlinear model (1) including the computation of the moments (6) serves as simulated reality. The simulated moments are used to provide the state feedback for the MPC. Note that there exists a considerable mismatch between the simulated reality and the linearized prediction model (11b). Thus we choose a prediction horizon of $N=13$. The control horizon, i.e. the number of varying values for each element of the input vector $u \in \mathcal{U} \subset \mathbb{R}^{2}$, equals the prediction horizon. This choice gives a good compromise between the numerical efforts to solve (12) and the closed-loop performance of the MPC. The weight matrices $Q$ and $R$ are specified as block diagonal matrices $Q=\operatorname{diag}\left(Q_{1}, Q_{2}\right), R=\operatorname{diag}\left(R_{1}, R_{2}\right)$. The blocks are $Q_{1}=\operatorname{diag}(2,2), Q_{2}=\operatorname{diag}(0,0,2), R_{1}=0.01, R_{2}=$ 0.1 . $^{2}$ The terminal penalty matrix is chosen as $P=Q$. The weight matrices $Q, R, P$ are chosen heuristically. The input box constraints (2) are $\bar{u}_{1}=0.5, \bar{u}_{2}=0.15$.

The plots in Fig. 6 show the simulation results for the uncontrolled system and for a previously developed FIR feedback filter, see [15] for details on the FIR feedback design. The upper left part of the figure depicts the bunch mean $\rho_{\varphi}$ over time. The upper right part shows bunch length $\mu_{\varphi}$ over time. The plots in the lower part of Fig. 6 show the corresponding input signals. The grey lines in Fig. 6 show the system response driven by the disturbance sequence (7) without control (i.e., $u_{1}(k)=u_{2}(k)=0$ for all $k \in \mathbb{N}$ ), the black dash-dot lines depict the result corresponding to the

\footnotetext{
${ }^{2}$ We merely penalize $\mu_{\varphi}$ via $Q_{2}$ since $\mu_{\omega}$ and $\mu_{\varphi}$ have linearly dependent steady state values, see (10).
} 
FIR filter. At time $k=368$ the disturbance $d_{2}(k)$ (7) hits the system. This leads to a jump of the bunch mean from a value close to 0 to 0.7 , see Fig. 6 upper left. Additionally, this disturbance induces oscillations of the bunch length in Fig. 6 upper right. Thus in the simulated reality, which is a good approximation of the real particle bunch dynamics, there exists a coupling between the dynamics of bunch length and bunch mean. In the prediction model used for the MPC controller (9) both dynamics are decoupled. Thus the (simulated) MPC controller is subject to plant-model mismatch. ${ }^{3}$

Fig. 7 shows the simulation results for the simulation MPC controller. The upper left part of the figure depicts the bunch mean $\rho_{\varphi}$ over time. The upper right part shows bunch length $\mu_{\varphi}$ over time. The plots in the lower part of Fig. 7 show the corresponding input signals. The black dash-dot lines show the results for the MPC scheme when the QP is solved up to a truncation error of $\epsilon=10^{-6}$, cf. (13). This means that in the (cold-started) FGM we do as many iterations as given by the upper bound presented below in (15). Considering the weight matrices and constraints as listed above this bound gives a maximum number of FGM iterations $i_{\max }=33$ required to achieve the desired tolerance of $\epsilon=10^{-6}$. Finally, the grey lines in Fig. 7 correspond to the solutions obtained with 15 iterations of the (cold-started) FGM.

Comparing the uncontrolled solutions with the FIR filter (Fig. 6) and the MPC schemes (Fig. 7) several observations can be made. The MPC schemes reduce the disturbanceinduced oscillations more effectively than the FIR filter. Additionally, none of the controllers achieve offset-free control, since the setpoint $\mu_{\varphi}=1$ is not attained. Finally, one should note that the differences between the inputs obtained with 15 FGM iterations $\left(\mathrm{MPC}_{i=15}\right)$ and the ones obtained with 33 iterations $\left(\mathrm{MPC}_{i=i_{\max }}\right)$ are hardly visible, cf. zoomedin plots in Fig. 7. This is in agreement with previous observations that the theoretical maximum iteration bound below in (15) is conservative, see [26].

\section{B. Performance Comparison}

As sketched in Section II-B the primary feedback goal is to keep the emittance small, i.e. the area occupied by the particle bunch in phase space. As the emittance itself cannot be measured in real-time directly, the oscillations of the bunch center $\rho_{\varphi}$ and length $\mu_{\varphi}$ are considered as controlled variables to indirectly decrease the emittance. For a performance comparison however, the emittance itself has to be evaluated after the simulation or the measurement.

The emittance increase of the open and closed loop is shown in Fig. 8. This clearly demonstrates that the MPC scheme performs better than the FIR filter. For the open loop, the emittance increases by about $80 \%$, the FIR filter reduces this to about 50\% and the MPC scheme to about $40 \%$. In particular, the MPC scheme drastically reduces the

\footnotetext{
${ }^{3}$ Obviously, the MPC could be reformulated as an offset-free design, cf. [20]. However, here we are interested in investigating the principle applicability of MPC to the longitudinal beam control problem. Thus offsetfree MPC is beyond the scope of this paper.
}

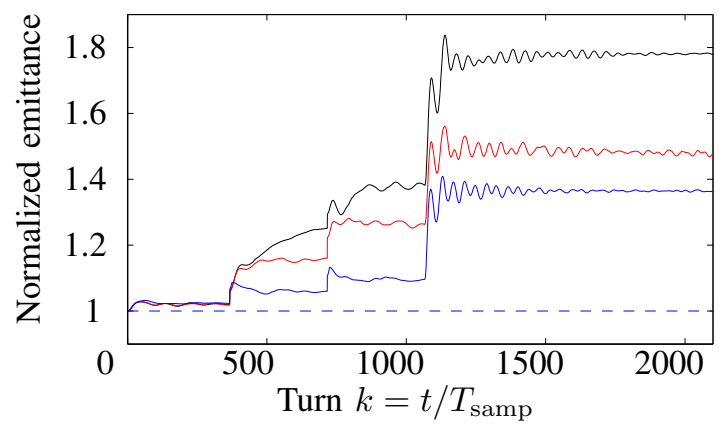

Fig. 8. Normalized emittance increase for the open loop (black), the FIR filter (red), and the MPC $(i=15)$ (blue).

emittance increase due to the disturbance $d_{1}$ : at $k=1068$, the emittance increase for the MPC is roughly $10 \%$, whereas it is about $30 \%$ and $40 \%$ for the FIR and the open-loop case, respectively.

At this point it is fair to ask the following questions: (a) Can we achieve the envisioned control performance of MPC in practice? (b) Can the required state information be reconstructed sufficiently accurately from real measurement data? And (c), is it possible to solve the QP (12) sufficiently quickly such that a sampling rate of $375 \mathrm{kHz}$ is feasible? While issues a) and b) are beyond the scope of this paper, we will sketch a preliminary answer to c) next.

\section{Real-time FeAsible FPGA Implementation}

The FGM is a gradient method which is efficiently applicable to convex nonlinear programming problems provided that the considered constraints have a rather simple geometry [18]. In practice, one can perform only a finite number of iterations of any optimization algorithm. This means that the iterate $z^{i}$ obtained at the final iteration $i=i_{\max }$ is only suboptimal; i.e.,

$$
\epsilon \geq J\left(z^{i_{\max }}\right)-J\left(z^{*}\right),
$$

where $\epsilon>0$ is the truncation error.

Denote by $L, l>0$ the largest and the smallest eigenvalue of the Hessian matrix of (12) and let

$$
c=\frac{\sqrt{L}-\sqrt{l}}{\sqrt{L}+\sqrt{l}} \text {. }
$$

The FGM can be summarized as follows:

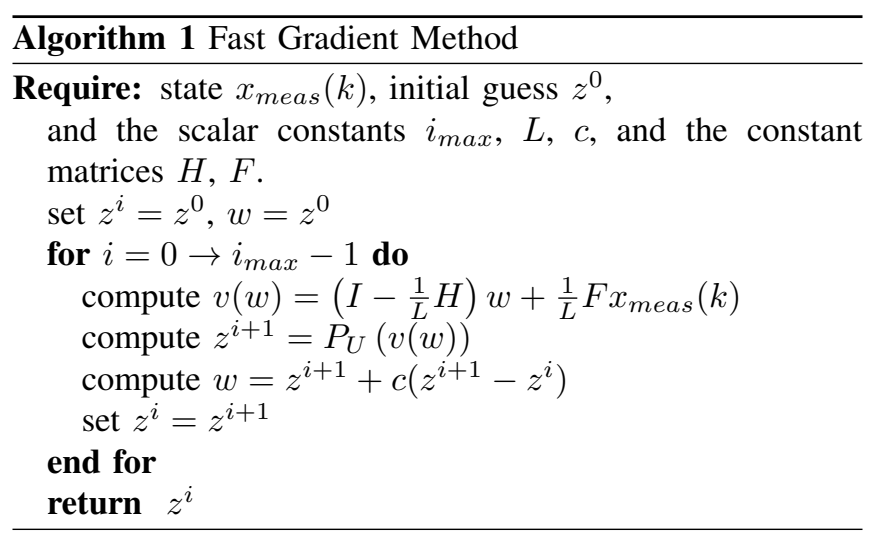


The function $P_{U}: \quad v \in \mathbb{R}^{\operatorname{dim}(u) \cdot N} \rightarrow \mathcal{U} \times \mathcal{U} \times \cdots \times \mathcal{U} \subset$ $\mathbb{R}^{\operatorname{dim}(u) \cdot N}$ denotes the projection onto the feasible set. This projection is extremely simple (element-wise saturation) in the case of box constraints, cf. [12, 21].

In addition to its low computational burden, only vector matrix multiplications and additions have to be performed online, the FGM admits an upper bound on the required number of iterations $i_{\max }$ in order to achieve a truncation error $\epsilon$, [19]. In the case of cold-starting $i_{\max }$ is given by

$$
i_{\max }=\min \left\{\left\lceil\frac{\ln 2 \epsilon-\ln (L+l) d^{2}}{\ln \left(1-\sqrt{\frac{l}{L}}\right)}\right\rceil,\left\lceil\sqrt{\frac{2(L+l) d^{2}}{\epsilon}}-2\right\rceil\right\},
$$

where $[\cdot]$ denotes rounding up to the next integer and the cold start initial guess is $u^{0}=(0,0, \ldots, 0)^{T} \in \mathbb{R}^{\operatorname{dim}(u) \cdot N}$. For the symmetric box constraints from (2) the constant $d$ is given by

$$
d=N \sum_{i=1}^{\operatorname{dim}(u)} \bar{u}_{i}^{2}
$$

The remaining question is whether or not Algorithm 1 can be computed sufficiently quickly to meet the required sampling period of $2.66 \mu \mathrm{s}$. The FIR filter currently used to control the SIS18 synchrotron [15] is implemented on a digital signal processor (DSP) on a custom-designed board described in [11]. The FIR filter already stretches the capabilities of the DSP and so we do not anticipate that the DSP will be an appropriate implementation technology for the FGM. However, the hardware described in [11] also includes a high-end FPGA, allowing the possibility of a custom hardware implementation of Algorithm 1. Consequently, we implemented the FGM of Algorithm 1 in a hardware description language, VHDL, and performed timing simulations using the TimeQuest Timing Analyzer that is provided as part of the Altera suite of FPGA design tools.

A fixed point arithmetic implementation of the FGM was developed based on the need for fast and simple arithmetic operations. An analysis as in [9, Proposition 1] was performed given the problem data to arrive at the need for three integral bits plus a sign bit.

A detailed analysis of the numerical requirements for the FGM was performed in [9], in particular with regards to the number of fractional bits required for a given set of problem data. In [9], several conditions on the fixed point problem were provided which must be checked after the design. These conditions on the fixed point data ensure the applicability of the FGM (by guaranteeing strong convexity of the objective function), ensuring numerical stability of Algorithm 1 in the presence of round-off errors, and guaranteeing the validity of the bound (15). Using 16 fractional bits with the problem data of the previous section satisfies all the conditions presented in [9].

The circuit diagram of our VHDL implementation is as shown in Figure 9. This single-cycle design requires significant on-chip resources due to the large number of arithmetic operations inherent in the matrix and constant mul-

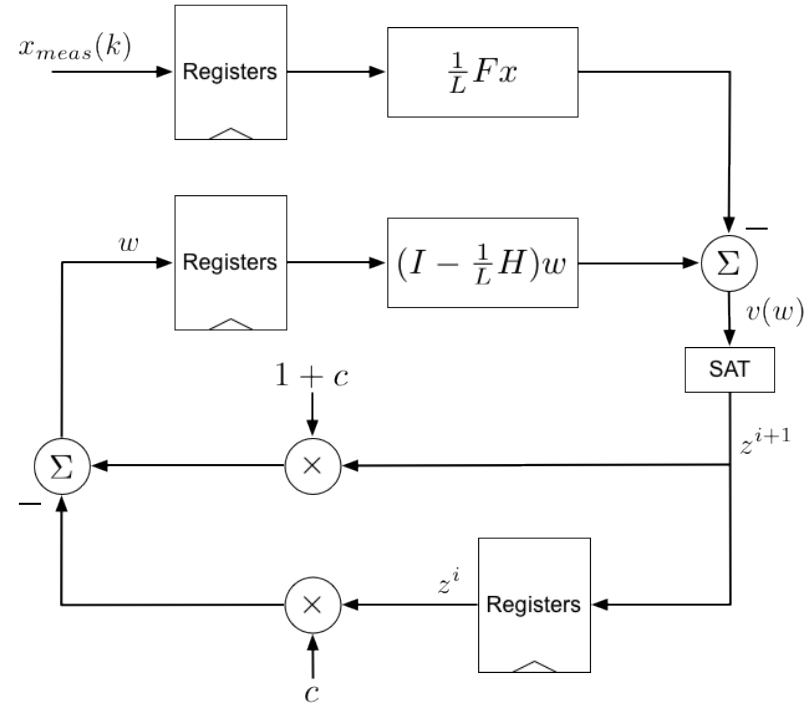

Fig. 9. VHDL Circuit Diagram.

tiplications, which necessitates implementation on a highend FPGA such as the Stratix V from Altera. Using the timing information for the Stratix $\mathrm{V}$ provided by Altera, the TimeQuest Timing Analyzer was used to determine a maximum allowable clock frequency of $29.9 \mathrm{MHz}$. Noting that in the circuit of Figure 9 one FGM iteration is performed per clock cycle, we see that the proposed 15 FGM iterations described in the previous section require $0.5 \mu \mathrm{s}$, placing the implementation well under the $2.66 \mu$ s sampling constraint. Furthermore, with a maximum number of FGM iterations of 33 taking $1.09 \mu \mathrm{s}$, performing the maximum number of iterations is possible in the event that this is required.

We emphasize that this is an initial hardware design for the purpose of demonstrating feasibility of implementing a model predictive controller for the SIS18 synchrotron. As such, many refinements of the design are possible in order to decrease latency and improve on-chip resource utilization by tuning the optimization settings within the Altera Quartus software, by performing a lower-level design on key components, and by exploiting available structure with the Hessian matrix.

Finally, we note that the main computational burden does not come from the number of states in the system model, $n$, but rather from the number of inputs, $m$ and the horizon length, $N$, of the optimization problem. This follows from the fact that in each FGM iteration it is necessary to perform the $N \cdot m$ by $N \cdot m$ matrix-vector multiplication. On the other hand, an increase in the state dimension has the effect of increasing the size of the matrix $F$, but it is only necessary to compute the product $F x$ once. Additionally, as is clear from Figure 9, in the first iteration the product $\frac{1}{L} F x$ is computed in parallel with $\left(I-\frac{1}{L} H\right) w$, which implies that as long as $n<N \cdot m$ it is the computation of the latter that is the critical element, even for the initial iteration. Consequently, we observe that the limiting factor in this design is the $26 \times 26$ matrix-vector multiplication. 


\section{CONCLUSIONS}

In this paper we presented a predictive control approach to longitudinal beam feedback control in hadron synchrotrons. We applied an MPC scheme whereby the quadratic problem is solved via a fast gradient method. Preliminary results indicate that the FGM is well-suited for implementation on an FPGA and sampling rates in the order of $1 \mathrm{MHz}$ are achievable. Future work will consider the effect of warmstarting the FGM on the FPGA, the implementation of a coupled observer-MPC scheme on the FPGA, hardwarespecific algorithm optimizations, and implementation on the real system.

\section{ACKNOWLEDGMENT}

The authors gratefully acknowledge the helpful comments on fast gradient methods by Markus Kögel from the Institute for Automation Engineering, Otto-von-Guericke-University Magdeburg.

\section{REFERENCES}

[1] N.P. Abreu, M. Blaskiewicz, J.M. Brennan, and C. Schultheiss. "Feedback damper system for quadrupole oscillations after transition at RHIC". In: Proceedings of the European Particle Accelerator Conference 2008 (EPAC08), Genoa, Italy. 2008.

[2] H. Damerau, M. Paoluzzi S. Hancock, M. Migliorati, and L. Ventura. "Longitudinal coupled-bunch oscillation studies in the CERN PS". In: Proceedings of the International Particle Accelerator Conference (IPAC13), Shanghai, China. 2013.

[3] S. Gayadeen and S. Duncan. "Anti-windup compensation for electron beam stabilisation control systems on synchrotrons with rate constrained actuators". In: Proceedings of the 2013 European Control Conference, Zürich, Switzerland. 2013, pp. 2752-2757.

[4] S. Gayadeen, S.R. Duncan, and W.P. Heath. "Design of multi-array controllers for electron beam stabilisation on synchrotrons". In: Proceedings of the 2013 American Control Conference, Washington DC, USA. 2013, pp. 1201-1206.

[5] S. Gering, J. Grieser, and A. Wahrburg. "Using LMIs to optimize robustness of observer-based state-feedback for a synchrotron". In: Int. J. Robust Nonlinear Control (2013).

[6] GSI Helmholtz Centre for Heavy Ion Research in Darmstadt, Germany. www.gsi.de.

[7] E.N. Hartley and J.M. Maciejowski. "Field programmable gate array based predictive control system for spacecraft rendezvous in elliptical orbits". In: Optimal Control Applications and Methods (2014).

[8] S.V. Ivanov and Q.P. Lebedev. "Analysis of the feedbackloop stability in the RF system of the U-70 synchrotron". In: Instruments and Experimental Techniques 45:5 (2002), pp. 6-19.

[9] J.L. Jerez, P.J. Goulart, S. Richter, G.A. Constantinides, E.C. Kerrigan, and M. Morari. "Embedded predictive control on an fpga using the fast gradient method". In: Proceedings of the 2013 European Control Conference, Zürich, Switzerland. 2013, pp. 3614-3620.

[10] H. Klingbeil, B. Zipfel, M. Kumm, and P. Moritz. "A digital beam-phase control system for heavy-ion synchrotrons". In: IEEE Transactions on Nuclear Science 54:6 (2007), pp. 2604-2610.
[11] H. Klingbeil, U. Laier, K.-P. Ningel, S. Schäfer, C. Thielmann, and B. Zipfel. "New digital low-level RF system for heavy-ion synchrotrons". In: Phys. Rev. ST Accel. Beams 14:10 (2011).

[12] M. Kögel and R. Findeisen. "A fast gradient method for embedded linear predictive control". In: Proceedings of the 18th IFAC World Congress, Milano, Italy. 2011, pp. 13621367.

[13] C.H. Kuo et al. "FPGA-based longitudinal bunch-by-bunch feedback system for TLS". In: Proceedings of the European Particle Accelerator Conference 2006 (EPAC06), Edinburgh, Scotland. 2006.

[14] S.Y. Lee. Accelerator Physics. World Scientific Pub. Co., 2004.

[15] D. Lens and H. Klingbeil. "Stability of longitudinal bunch length feedback for heavy-ion synchrotrons". In: Phys. Rev. ST Accel. Beams 16:3 (2013), p. 032801.

[16] K.V. Ling, B.F. Wu, and J.M. Maciejowski. "Embedded model predictive control (MPC) using a FPGA". In: Proc. 17th IFAC World Congress, Seoul, South Korea. 2008, pp. 15250-15255.

[17] A. Napier, S. Gayadeen, and S. R. Duncan. "Fast orbit beam stabilisation for a synchrotron". In: Proceedings of the 2011 IEEE International Conference on Control Applications. Denver, CO, USA, 2011, pp. 1094-1099.

[18] Y. Nesterov. "A method of solving a convex programming problem with convergence rate O (1/k2)". In: Soviet Mathematics Doklady 27:2 (1983), pp. 372-376.

[19] Y. Nesterov. Introductory Lectures on Convex Optimization. A Basic Course. Vol. 87. Springer, 2004.

[20] J.B. Rawlings and D.Q. Mayne. Model Predictive Control: Theory \& Design. Nob Hill Publishing, Madison, WI, 2009.

[21] S. Richter, C.N. Jones, and M. Morari. "Real-time inputconstrained MPC using fast gradient methods". In: Proceedings of the 48th IEEE Conference on Decision and Control and the 28th Chinese Control Conference, CDC/CCC, Shanghai, China. 2009, pp. 7387-7393.

[22] S. Sakanaka and T. Obina. "Analysis of the feedback system used to damp longitudinal quadrupole-mode bunch oscillations". In: Proceedings of the 2003 Particle Accelerator Conference (PAC). 2003, pp. 3365-3367.

[23] A.H. Sørensen. "Liouville's theorem and emittance". In: CAS - CERN Accelerator School, 3rd general accelerator physics course. 1988.

[24] H. Stoecker and C. Sturm. "The FAIR start". In: Nuclear Physics A 855:1 (2011), pp. 506-509.

[25] A.G. Wills, G. Knagge, and B. Ninness. "Fast linear model predictive control via custom integrated circuit architecture". In: IEEE Transactions on Control Systems Technology 20:1 (2012), pp. 59-71.

[26] P. Zometa, M. Kögel, T. Faulwasser, and R. Findeisen. "Implementation aspects of model predictive control for embedded systems". In: Proceedings of the 2012 American Control Conference, Montreal, Canada. 2012, pp. 12051210 . 